\title{
The Configuration Space of Regular Spherical Even Polygons
}

\author{
Yasuhiko Kamiyama \\ Department of Mathematics, University of the Ryukyus, Nishihara-Cho, Okinawa 903-0213, Japan \\ Correspondence should be addressed to Yasuhiko Kamiyama; kamiyama@sci.u-ryukyu.ac.jp
}

Received 27 September 2019; Accepted 28 October 2019; Published 14 November 2019

Academic Editor: Vladimir V. Mityushev

Copyright (C) 2019 Yasuhiko Kamiyama. This is an open access article distributed under the Creative Commons Attribution License, which permits unrestricted use, distribution, and reproduction in any medium, provided the original work is properly cited.

Let $a$ be a real number satisfying $0<a<\pi$. We denote by $M_{n}(a)$ the configuration space of regular spherical $n$-gons with side lengths $a$. In our previous paper, we determined $\chi\left(M_{n}(a)\right)$ for odd $n$. In this paper, we determine it for even $n$. The main difference from our previous paper is given as follows. When $n$ is odd, we prove that $M_{n}(a)$ is obtained from $S^{n-3}$ by successive Morse surgeries. On the other hand, when $n$ is even, we show that $M_{n}(a)$ is obtained from $\mathscr{P}_{n}$ by successive Morse surgeries. Here, $\mathscr{P}_{n}$ denotes the configuration space of equilateral $n$-gons in $\mathbb{R}^{2}$, which has singular points when $n$ is even.

\section{Introduction and Statement of the Main Result}

Recently, the topology of polygon spaces in the Euclidean space of dimension two or three has been considered by many authors. The study of planar polygon spaces started in [1-3]. For example, the homology groups were determined in [4]. On the other hand, the study of spatial polygon spaces started in [5].

Morse theory plays a key role in the study of polygon spaces. [6] is an excellent exposition about polygon spaces with emphasis on Morse theory. In [7], Milgram and Trinkle obtained results by making excellent use of Morse surgery.

Later, Kapovich and Millson [8] studied spherical polygon spaces. They proved a remarkable and very useful theorem about a Morse function. We first recall their result.

Let $r=\left(r_{1}, r_{2}, \ldots, r_{n}\right)$ be an $n$-tuple of real numbers satisfying $0<r_{i}<\pi$. We set $r^{\prime}:=\left(r_{1}, \ldots, r_{n-1}\right)$ and define

$$
\tilde{N}_{r^{\prime}}=\left\{u=\left(u_{1}, \ldots, u_{n}\right) \in\left(S^{2}\right)^{n} \mid d\left(u_{i}, u_{i+1}\right)=r_{i}, 1 \leq i \leq n-1\right\},
$$

where $d$ is the spherical distance. Let $S O(3)$ act on $\tilde{N}_{r^{\prime}}$ diagonally and we set $N_{r^{\prime}}:=\widetilde{N}_{r_{1}} / S O(3)$. Kapovich and Millson defined the function $\rho_{n}: N_{r^{\prime}} \longrightarrow \mathbb{R}$ by

$$
\rho_{n}(u)=d\left(u_{1}, u_{n}\right) \text {. }
$$

We will restrict to $u$ 's such that $0<\rho_{n}(u)<\pi$ so that $\rho_{n}$ is differentiable. Note that

$$
M_{r}:=\rho_{n}^{-1}\left(r_{n}\right) \subset N_{r^{\prime}},
$$

is the moduli space of closed polygonal linkages in $S^{2}$ with side lengths $\left(r_{1}, \ldots, r_{n}\right)$.

It is proved in [8] that an element of $N_{r}^{\prime}$ is a critical point of $\rho_{n}$ if and only if it is degenerate, i.e., it lies in a great circle $\gamma$ in $S^{2}$. In order to describe the signature, we give the following.

Definition 1 (orienting $\gamma$ ). Suppose $u=\left(u_{1}, u_{2}, \ldots, u_{n}\right)$ is a closed degenerate linkage contained in a great circle $\gamma$. Orient $\gamma$ so that the arc joining $u_{1}$ to $u_{n}$ is positively directed. Thus, an edge $e_{i}$ is a back-track if it has the same direction as $e_{n}=\left(u_{n}, u_{1}\right)$.

Theorem 1 ([8], Main Theorem). Let $T \in N_{r}^{\prime}$ be a degenerate free $(n-1)$ linkage and $P$ be the associated degenerate closed n-gon linkage. Then, the signature of $\left.D^{2} \rho_{n}\right|_{T}$ is given by

$$
(b(P)+2 w(P)-1, f(P)-2 w(P)-1),
$$

where $f=f(P)$ denotes the number of forward-tracks and $b=b(P)$ denotes the number of back-tracks so $f+b=n$. 
Moreover, $w(P)$ denotes the winding number of the degenerate configuration $P$, which is given by the formula $\sum_{i=1}^{n} \varepsilon_{i} r_{i}=2 \pi w(P)$, where $\varepsilon_{i} \in\{ \pm 1\}$ is defined as follows: if $e_{i}$ is the forward-track, the we set $e_{i}=1$, and if $e_{i}$ is the backtrack, then we set $e_{i}=-1$.

Let $a$ be a real number satisfying $0<a<\pi$. We consider the case that $r=(a, a, \ldots, a)$, that is, $r_{i}=a$ for $1 \leq i \leq n$. We set

$$
M_{n}(a):=\rho_{n}^{-1}(a)
$$

Note that $M_{n}(a)$ is the moduli space of regular spherical polygons with side lengths $a$.

We realize $M_{n}(a)$ as a level set of a Morse function, which is different from the above $\rho_{n}$. We first set

$$
\begin{aligned}
A_{n} & :=\left\{(P, a)=\left(\left(u_{1}, \ldots, u_{n}\right), a\right) \in\left(S^{2}\right)^{n} \times(0, \pi) \mid d\left(u_{i}, u_{i+1}\right)\right. \\
& \left.=a \text { for } 1 \leq i \leq n-1 \text { and } d\left(u_{n}, u_{1}\right)=a\right\} .
\end{aligned}
$$

Let $S O(3)$ act on $A_{n}$ by

$$
(P, a) g:=(P g, a)
$$

where $g \in S O(3)$. Then, we set

$$
X_{n}:=\frac{A_{n}}{S O(3)}
$$

We define the function $\mu: X_{n} \longrightarrow \mathbb{R}$ by

$$
\mu(P, a)=a \text {. }
$$

Note that for all $a \in(0, \pi)$, we have

$$
\mu^{-1}(a)=M_{n}(a) \text {. }
$$

In [9], we studied the case of odd $n$ and proved that $\mu$ is a Morse function. Since a level set is obtained by successive Morse surgeries, we could determine $\chi\left(M_{n}(a)\right)$ for all $a$.

The purpose of this paper is to study the case of even $n$. Hereafter, we will always assume $n$ to be even and set $n=2 m$. When $n$ is even, $M_{n}(a)$ has the following two characteristics, which do not hold for odd $n$. One is that there is a homeomorphism $M_{n}(a) \cong M_{n}(\pi-a)$ for all $a$ (see Theorem 5 (i)). The other is that $M_{n}(a)$ has singular points for all $a$. In fact, we set

$$
\Sigma_{n}(a):=\left\{P \in M_{n}(a) \mid P \text { is degenerate such that } w(P)=0\right\},
$$

where $w(P)$ is defined in Theorem 1. Then, an element of $\Sigma_{n}(a)$ is a singular point of $M_{n}(a)$.

Avoiding $\Sigma_{n}(a)$, we give the following definitions. First, we set

$$
\bar{M}_{n}(a):=M_{n}(a) \backslash \Sigma_{n}(a) .
$$

Second, we set

$$
\bar{X}_{n}:=\left\{(P, a) \in X_{n} \mid P \in \bar{M}_{n}(a)\right\} .
$$
to $\bar{X}_{n}$
Theorems 2 and 3, which are main theorems of this paper, assert that $\bar{\mu}$ is a Morse function.

Theorem 2. An element $(P, a) \in \bar{X}_{n}$ is a critical point of $\bar{\mu}$ if and only if $P$ is degenerate.

Theorem 2 implies the following two results:

(i) In contrast to the fact that the critical points of $\rho_{n}$ are the degenerate linkages, the critical points of $\bar{\mu}$ are the degenerate linkages $P$ such that $w(P) \neq 0$.

(ii) All $a$ 's which are critical values for $\bar{\mu}$ are rational multiples of $\pi$. Moreover, the smallest critical value is $\pi / m$ and the largest one is $(m-1) \pi / m$.

From the definition of $f, b$, and $w$ in Theorem 1 , for $P \in M_{n}(a)$ a degenerate linkage, the following relations hold:

$$
\left\{\begin{array}{l}
f+b=n, \\
a(f-b)=2 \pi w .
\end{array}\right.
$$

Then, we have the following:

Theorem 3. Let $P \in \bar{M}_{n}(a)$ be a degenerate polygon. Then, the following hold:

(i) When $w(P)>0$, the signature of $D^{2} \bar{\mu}_{(P, a)}$ is given by $(f(P)-2 w(P)-1, b(P)+2 w(P)-1)$

(ii) When $w(P)<0$, the signature of $\left.D^{2} \bar{\mu}\right|_{(P, a)}$ is given by $(b(P)+2 w(P)-1, f(P)-2 w(P)-1)$

This paper is organized as follows. In Section 2, we first combine Theorems 2 and 3 into Theorem 4 . Then, using the theorem, we determine $\chi\left(M_{n}(a)\right)$ in Theorem 6. In Section 3 , we prove Theorems 2 and 3. Theorem 1 is a key to proving Theorem 3. In Section 4, we give several examples about $\chi\left(M_{n}(a)\right)$.

\section{Conclusions from Theorems 2 and 3}

In Theorem 3, we set

$$
(s, t):=\left\{\begin{array}{cc}
\left(\frac{f(P)-b(P)}{2}, w(P)\right), & \text { if } w(P)>0, \\
\left(\frac{-f(P)+b(P)}{2},-w(P)\right), & \text { if } w(P)<0 .
\end{array}\right.
$$

Then, we obtain the following theorem. We denote by $\mathbb{N}=\{1,2,3, \ldots$,$\} the set of natural numbers.$

Theorem 4. We set

$$
\Gamma_{n}:=\{(s, t) \in \mathbb{N} \times \mathbb{N} \mid t<s \leq m\} .
$$

Then, the following assertions hold:

(i) To each $(s, t) \in \Gamma_{n}$, there corresponds a certain number of critical points of $\bar{\mu}$. All critical points are nondegenerate such that their information is given by Table 1. 
TABLE 1: The information on $\bar{\mu}$ at $(s, t)$.

\begin{tabular}{lcc}
\hline Critical value & The number of critical points & The index \\
\hline$(t / s) \pi$ & $\left(\begin{array}{c}2 m \\
m-s\end{array}\right)$ & $m-s+2 t-1$ \\
\hline
\end{tabular}

(ii) Conversely, a critical point of $\bar{\mu}$ is attained by a unique $(s, t) \in \Gamma_{n}$.

\section{Remark 1}

(i) It is not true that critical points of the same critical value have the same index. For example, $\Gamma_{12}$ contains elements $(3,1)$ and $(6,2)$ such that their critical values are $\pi / 3$. On the other hand, the index of the former is 4 but that of the latter is 3 .

(ii) The number of critical points does not depend on $t$. Moreover, the index of $\bar{\mu}$ has the same parity as $m-s-1$, which also does not depend on $t$.

Proof of Theorem 4

(i) Let $U_{n}$ be the set of critical points of $\bar{\mu}$. We define the map

$$
F: U_{n} \longrightarrow \Gamma_{n}
$$

by making $(P, a) \in U_{n}$ correspond with $(s, t)$ in (15). We check that $F$ is certainly a map to $\Gamma_{n}$. First, we check that $t>0$. In fact, this is clear from (15) for $w(P)$ positive or negative. Second, we check that $t<s$. In fact, we have from the second equation of (14) that

$$
a s=\pi t .
$$

Since $0<a<\pi$, (18) implies that $t<s$. Third, we check that $s \leq m$. In fact, we have from the second equation of (15) that if $w(P)>0$, then $s \leq f / 2 \leq m$, and if $w(P)<0$, then $s \leq b / 2 \leq m$.

Next, we check Table 1. First, (18) tells us that the critical value in Table 1 is true.

Second, we compute the index of $\bar{\mu}$ at $(s, t)$. From the first equation of (14) and (15), we have

$$
(f, b)= \begin{cases}(m+s, m-s), & \text { if } w>0, \\ (m-s, m+s), & \text { if } w<0 .\end{cases}
$$

Then, using Theorem 3, (15), and (19), we have the index of $\bar{\mu}$ at $(s, t)$ is $m-s+2 t-1$ for $w$ positive or negative. Hence, the index of $\bar{\mu}$ in Table 1 is true.
Third, we compute the number of critical points. (15) tells us that critical points $(P, a)$ and $(Q, a)$ satisfy $F(P, a)=F(Q, a)$ if and only if $(f(P)$, $b(P))=(f(Q), b(Q)) \quad$ or $\quad(f(P), b(P))=(b(Q)$, $f(Q))$. The description of $f$ in terms of $s$ is computed in (19). Since $e_{n}$ is always back-track, we need to choose $f$ elements from $\left\{e_{1}, e_{2}, \ldots, e_{2 m-1}\right\}$. The total number of such choices is

$$
\begin{aligned}
\left(\begin{array}{c}
2 m-1 \\
m+s
\end{array}\right)+\left(\begin{array}{c}
2 m-1 \\
m-s
\end{array}\right) & =\left(\begin{array}{c}
2 m-1 \\
m-s-1
\end{array}\right)+\left(\begin{array}{c}
2 m-1 \\
m-s
\end{array}\right) \\
& =\left(\begin{array}{c}
2 m \\
m-s
\end{array}\right) .
\end{aligned}
$$

Hence, the number of critical points in Table 1 is true. This completes the proof of Theorem 4 (i).

(ii) The item is clear from (15).

\section{Theorem 5}

(i) For $r=\left(r_{1}, r_{2}, \ldots, r_{n}\right)$, we set $r^{\prime}:=\left(r_{1}^{\prime}, r_{2}^{\prime}, \ldots, r_{n}^{\prime}\right)$, where we put $r_{i}^{\prime}:=\pi-r_{i}$ for $1 \leq i \leq n$. Then, there is a homeomorphism $M_{r} \cong M_{r^{\prime}}$, where the space $M_{r}$ is defined in (3). In particular, there is a homeomorphism $M_{n}(a) \cong M_{n}(\pi-a)$ for all $a \in(0, \pi)$.

(ii) Let $\mathscr{P}_{n}$ be the configuration space of equilateral $n$ gons in $\mathbb{R}^{2}$. Then, if $0<a<\pi / m$ or $(m-1) \pi / m<a<\pi$, there is a homeomorphism $M_{n}(a) \cong \mathscr{P}_{n}$.

Proof

(i) The item is proved in ([10], Theorem 2.2) and ([8], Section 4). More precisely, we define the map $G$ : $M_{r} \longrightarrow M_{r}$ by

$$
G\left(u_{1}, \ldots, u_{n}\right):=\left(v_{1}, \ldots, v_{n}\right),
$$

where we set $v_{i}:=(-1)^{i} u_{i}$. Then, $G$ is a homeomorphism.

(ii) It is clear that if $\varepsilon$ is a sufficiently small positive real number, then $M_{n}(\varepsilon)$ is homeomorphic to $\mathscr{P}_{n}$. Moreover, since any element of $(0, \pi / m)$ is a regular value of $\bar{\mu}$ by Theorem 4 , we have $M_{n}(a) \cong \mathscr{P}_{n}$ for $0<a<\pi / m$. Using the above item (i), we also have $M_{n}(a) \cong \mathscr{P}_{n}$ for $(m-1) \pi / m<a<\pi$.

Theorem 6. Let $V_{n}$ be the set of critical values of $\bar{\mu}$ (note that we can write the elements of $V_{n}$ using Table 1). Then, the following assertions hold: 
(i) Assume that $a \notin V_{n}$. Then, we have

$$
\chi\left(M_{n}(a)\right)=(-1)^{m+1}\left(\begin{array}{c}
2 m-1 \\
m
\end{array}\right)
$$

(ii) Assume that $a \in V_{n}$. Let $\left(s_{0}, t_{0}\right)$ be the unique element of $\Gamma_{n}$, which satisfies the following two conditions:

$$
\begin{aligned}
\operatorname{gcd}\left(s_{0}, t_{0}\right) & =1, \\
\frac{t_{0}}{s_{0}} \pi & =a .
\end{aligned}
$$

Then, we have

$\chi\left(M_{n}(a)\right)=(-1)^{m+1}\left\{\left(\begin{array}{c}2 m-1 \\ m\end{array}\right)+\sum_{i=1}^{\left\lfloor m / s_{0}\right\rfloor}(-1)^{s_{0} i}\left(\begin{array}{c}2 m \\ m-s_{0} i\end{array}\right)\right\}$.

Note that the right-hand side of (24) does not depend on $t_{0}$.

In order to prove Theorem 6, we need the following.

Theorem 7. Let $h: M \longrightarrow \mathbb{R}$ be a smooth function on a $d$-dimensional manifold $M$. For numbers $\xi_{1}<\xi_{2}$, we assume that $h^{-1}\left[\xi_{1}, \xi_{2}\right]$ is compact and contains a unique nondegenerate critical point $p$ of index $r$. Then, the following results hold:

(i) The level set $h^{-1}\left(\xi_{1}\right)$ is obtained from $h^{-1}\left(\xi_{2}\right)$ by removing Int $\left(\partial D^{d-r} \times D^{r}\right)$ and attaching $D^{d-r} \times \partial D^{r}$ along the boundary. We call this construction a surgery of type $(d-r, r)$.

(ii) If $d$ is even, then we have

$$
\chi\left(h^{-1}\left(\xi_{1}\right)\right)=\chi\left(h^{-1}\left(\xi_{2}\right)\right)
$$

(iii) We set $\xi_{3}:=h(p)$. Then, level set $h^{-1}\left(\xi_{3}\right)$ is obtained from $h^{-1}\left(\xi_{2}\right)$ by removing Int $\left(\partial D^{d-r} \times D^{r}\right)$ and attaching $C\left(S^{d-r-1} \times S^{r-1}\right)$ along the boundary, where $C$ denotes the cone. In particular, if $d$ is even, then we have

$$
\chi\left(h^{-1}\left(\xi_{3}\right)\right)=\chi\left(h^{-1}\left(\xi_{2}\right)\right)+(-1)^{r} .
$$

Proof. The theorem is well known in Morse surgery (see [11]).

Proof of Theorem 6. We apply Theorem 7 to the function $\mu$ in (9). Every level set $M_{n}(a)$ contains the singular point set $\Sigma_{n}(a)$ and the information on $\bar{\mu}$ is determined in Theorem 4.

(i) If $a \notin V_{n}$, then Table 1 tells us that $a$ is a regular value of $\bar{\mu}$. Combining Theorem 7 (ii) and Theorem
5 (ii), we have $\chi\left(M_{n}(a)\right)=\chi\left(\mathscr{P}_{n}\right)$. Recall that $\chi\left(\mathscr{P}_{n}\right)$ was determined in ([12], Theorem A). Hence, (i) follows.

(ii) If $a \in V_{n}$, then Table 1 tells us that the elements of $\Gamma_{n}$ whose critical value equals to $a$ are given by $\left(s_{0} i, t_{0} i\right)$ for $1 \leq i \leq\left\lfloor\mathrm{m} / \mathrm{s}_{0}\right\rfloor$. Then, combining Table 1 and Theorem 7 (iii), we obtain (ii).

\section{Proofs of Theorems 2 and 3}

Proof of Theorem 2. We prove the theorem along the lines of ([8], Theorem 2.9) (see also the proof of ([9], Theorem 1.3)). Combining the following three assertions, we obtain Theorem 2:

(i) An analogue of ([8], Lemma 2.7 (ii)): by (10), the Zariski tangent space $T_{P} \bar{M}_{n}(a)$ is given by

$$
T_{P} \bar{M}_{n}(a)=\left.\operatorname{ker} d \bar{\mu}\right|_{(P, a)} .
$$

(ii) An analogue of ([8], Corollary 2.8): we see from (i) that a point $P$ is a singular point of $\bar{M}_{n}(a)$ if and only if $(P, a)$ is a critical point of $\bar{\mu}$.

(iii) By ([13], Theorem 1.1), $P$ is a singular point of $\bar{M}_{n}(a)$ if and only if $P$ is degenerate.

In order to prove Theorem 3, we need the following.

Lemma 1. We consider the function $\rho_{n}$ in (2) for the case that $r^{\prime}=(a, a, \ldots, a)$, that is, $r_{i}=a$ for $1 \leq i \leq n-1$. Let $O$ be an open neighborhood of $(P, a)$ in $\bar{X}_{n}$ such that $O$ contains no other degenerate polygons than $(P, a)$. We fix a sufficiently small positive real number $\varepsilon$. Then, the following assertions hold:

(i) Assume that $w(P)>0$. Then, for all $j \in\{-1,1\}$, $\bar{\mu}^{-1}(a+j \varepsilon) \cap O$ is diffeomorphic to an open set of $\rho_{n}^{-1}(a-j \varepsilon)$.

(ii) Assume that $w(P)<0$. Then, for all $j \in\{-1,1\}$, $\bar{\mu}^{-1}(a+j \varepsilon) \cap O$ is diffeomorphic to an open set of $\rho_{n}^{-1}(a+j \varepsilon)$.

Proof of Lemma 1

(i) We write the side lengths of a spherical polygon as $\left(r_{1}, r_{2}, \ldots \ldots, r_{n}\right)$. For $\delta \in[0, \varepsilon]$, we deform the side lengths of an element of $\bar{\mu}^{-1}(a+\varepsilon) \cap O$ by

$$
(a+j \delta, a+j \delta, \ldots, a+j \delta, a+j(-\varepsilon+2 \delta)) .
$$

We need to check that this deformation is indeed possible. To see this, it will suffice to see that (28) does not cross a wall for any $\delta$, where a wall is defined in ([8], p. 311).

Recall that $e_{n}$ is always back-track (see Definition 1). Hence, we have about (28) that 
the sum of forward-track side-lengths - the sum of back

$$
\text { -track side-lengths }=2 \pi w(P)+j(\varepsilon+(u-2) \delta),
$$

for some integer $u$. We claim that $u \geq 3$. In fact, the assumption $w(P)>0$ tells us that at least $m+1$ of the first $2 m-1$ components of (28) are forward-track. Hence, we have $u \geq(m+1)-(m-2)=3$ and the claim follows.

Now, since the term $j(\varepsilon+(u-2) \delta)$ in (2) is near zero but not zero for all $\delta \in[0, \varepsilon],(28)$ does not cross a wall for any $\delta$. Finally, setting $\delta=0$ or $\varepsilon$ in (28), we complete the proof of (i).

(ii) Instead of (28), we deform the side lengths of an element of $\bar{\mu}^{-1}(a+\varepsilon) \cap O$ by

$$
(a+j \delta, a+j \delta, \ldots, a+j \delta, a+j \varepsilon) .
$$

We have about (30) that

the sum of forward-track side-lengths - the sum of back

$$
\text { -track side-lengths }=2 \pi w(P)-j(\varepsilon+v \delta),
$$

for some integer $v$. We claim that $v \geq 1$. In fact, the assumption $w(P)<0$ tells us that at most $m-1$ of the first $2 m-1$ components of (30) are forward-track. Hence, we have $-v \leq(m-1)-m=-1$ and the claim follows.

Now, since the term $j(\varepsilon+v \delta)$ in (9) is near zero but not zero for all $\delta \in[0, \varepsilon],(30)$ does not cross a wall for any $\delta$. Finally, setting $\delta=0$ or $\varepsilon$ in (30), we complete the proof of (ii). This completes the proof of Lemma 1 .

Proof of Theorem 3. We shall deduce Theorem 3 from Theorem 1. We apply Theorem 7 (i) to the map $\bar{\mu}$. Note that $\operatorname{dim} \bar{X}_{n}=n-2$. We denote by $r$ the index of $P$. When the level set $\bar{\mu}^{-1}(a+\varepsilon)$ descends to $\bar{\mu}^{-1}(a-\varepsilon)$, the critical point $P$ gives a surgery of type

$$
(n-2-r, r)
$$

We set $\lambda:=f(P)-2 w(P)-1$.

(i) If $w(P)>0$, then Lemma 1 (i) tells us that the above descent is equivalent to the ascent from $\rho_{n}^{-1}(a-\varepsilon)$ to $\rho_{n}^{-1}(a+\varepsilon)$. Combining Theorem 7 (i) and Theorem 1, when we cross through $P$, a surgery of type

$$
(\lambda, n-2-\lambda)
$$

occurs. Comparing (32) and (33), we have

$$
r=n-2-\lambda=b(P)+2 w(P)-1 .
$$

(ii) If $w(P)<0$, then the descent from $\bar{\mu}^{-1}(a+\varepsilon)$ to $\bar{\mu}^{-1}(a-\varepsilon)$ is equivalent to the descent from $\rho_{n}^{-1}(a+$ $\varepsilon)$ to $\rho_{n}^{-1}(a-\varepsilon)$. When we cross through $P$, a surgery of type

$$
(n-2-\lambda, \lambda)
$$

occurs. Comparing (32) and (35), we have

$$
r=\lambda=f(P)-2 w(P)-1 .
$$

This completes the proof of Theorem 3.

\section{Examples}

Proposition 1. We have the following examples:

(i) $\chi\left(M_{n}(\pi / 2)\right)=(-1)^{m+1} \cdot 2^{2 m-2}$

(ii) $\chi\left(M_{n}(\pi / 3)\right)=(-3)^{m-1}$

(iii) $\chi\left(M_{n}(\pi / 4)\right)=(-1)^{m+1} \cdot 2^{m-2} \cdot\left(2^{m-1}+1\right)$

(iv) $\chi\left(M_{n}(\pi / 6)\right)=(-1)^{m+1} \cdot\left(\left(4^{m-1} / 3\right)+\left(3^{m-1} / 2\right)+\right.$ $(1 / 6))$

Proof. Computing the right-hand side of (24) explicitly, we obtain the proposition.

Remark 2. Proposition 1 (i) was obtained in ([14], Theorem 2) by a different method.

\section{Data Availability}

No data were used to support this study.

\section{Conflicts of Interest}

The author declares that there are no conflicts of interest regarding the publication of this paper.

\section{Acknowledgments}

This work was supported by JSPS KAKENHI (grant no. 15K04877).

\section{References}

[1] J.-C. Hausmann, Sur la Topologie des bras Articulés, Lecture Notes in Mathematics 1474, Springer-Verlag, Berlin, Germany, 1989.

[2] M. Kapovich and J. Millson, "On the moduli space of polygons in the Euclidean plane," Journal of Differential Geometry, vol. 42, no. 2, pp. 430-464, 1995.

[3] K. Walker, "Configuration spaces of linkages," Undergraduate thesis, Princeton University, Princeton, NJ, USA, 1985.

[4] M. Farber and D. Schütz, "Homology of planar polygon spaces," Geometriae Dedicata, vol. 125, no. 1, pp. 75-92, 2007. 
[5] M. Kapovich and J. J. Millson, "The symplectic geometry of polygons in Euclidean space," Journal of Differential Geometry, vol. 44, no. 3, pp. 479-513, 1996.

[6] M. Farber, Invitation to Topological Robotics, Zurich Lectures in Advanced Mathematics, European Mathematical Society (EMS), Zürich, Switzerland, 2008.

[7] R. J. Milgram and J. C. Trinkle, "The geometry of configuration spaces for closed chains in two and three dimensions," Homology, Homotopy and Applications, vol. 6, no. 1, pp. 237-267, 2004.

[8] M. Kapovich and J. J. Millson, "On the moduli space of a spherical polygonal linkage," Canadian Mathematical Bulletin, vol. 42, no. 3, pp. 307-320, 1999.

[9] Y. Kamiyama, "The Euler characteristic of the regular spherical polygon spaces," Homology, Homotopy and Applications, vol. 22, no. 1, pp. 1-10, 2019, In press.

[10] A. Galitzer, The moduli space of polygon linkages in the 2sphere, Ph.D. thesis, University of Maryland, College Park, MD. USA, 1997.

[11] J. Milnor, Morse Theory: Annals of Mathematics Studies, Vol. 51, Princeton University Press, Princeton, NJ, USA, 1963.

[12] Y. Kamiyama, "Topology of equilateral polygon linkages," Topology and its Applications, vol. 68, no. 1, pp. 13-31, 1996.

[13] M. Kapovich and J. J. Millson, "Hodge theory and the art of paper folding," Publications of the Research Institute for Mathematical Sciences, vol. 33, no. 1, pp. 1-31, 1997.

[14] Y. Kamiyama, "A hypersurface of the configuration space of a spatial robot arm," JP Journal of Geometry and Topology, vol. 20 , no. 1, pp. 27-38, 2017. 


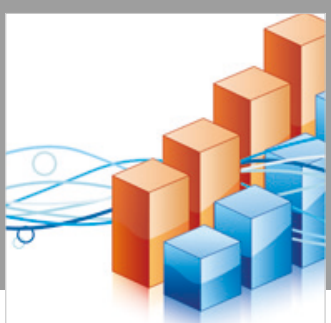

Advances in

Operations Research

\section{-n-m}
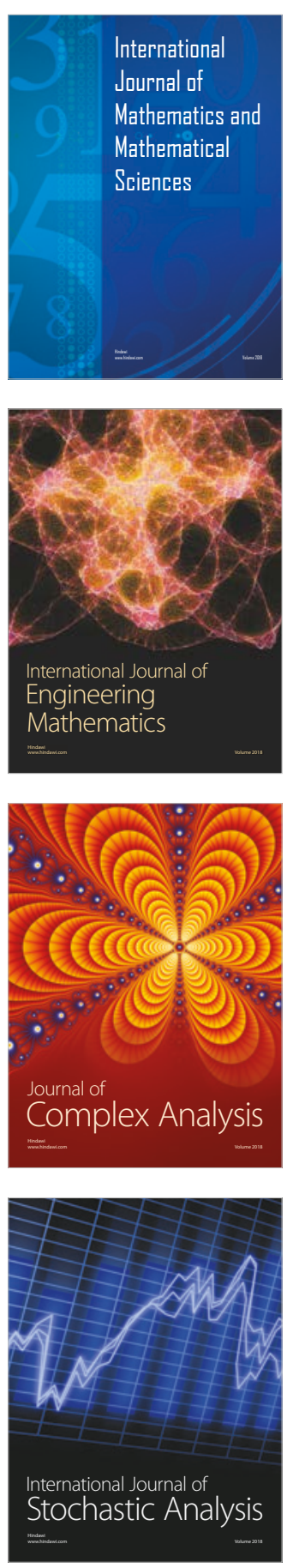
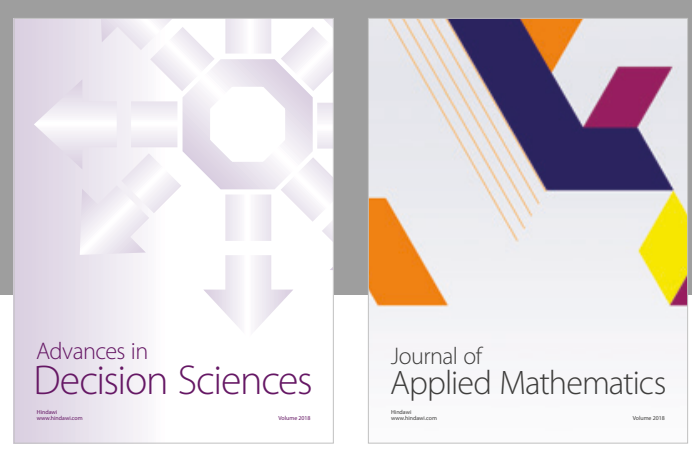

Journal of

Applied Mathematics
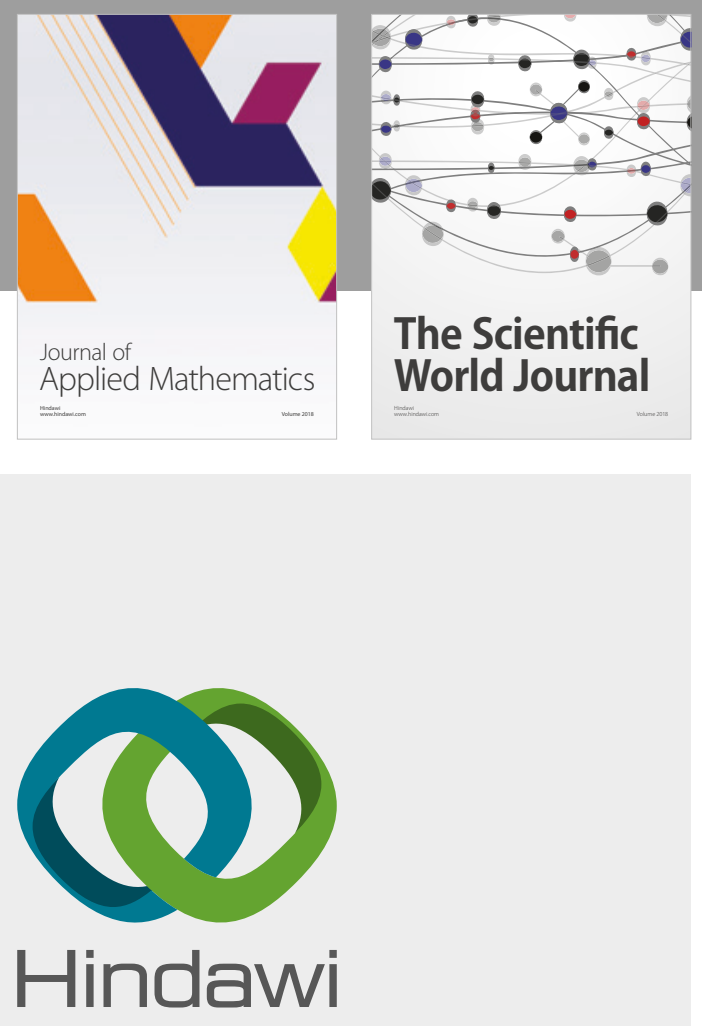

Submit your manuscripts at

www.hindawi.com

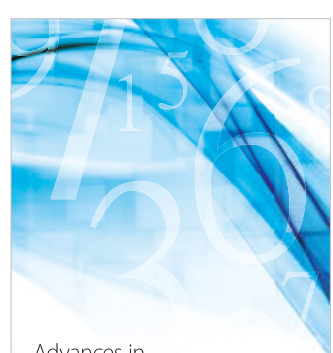

Advances in
Numerical Analysis
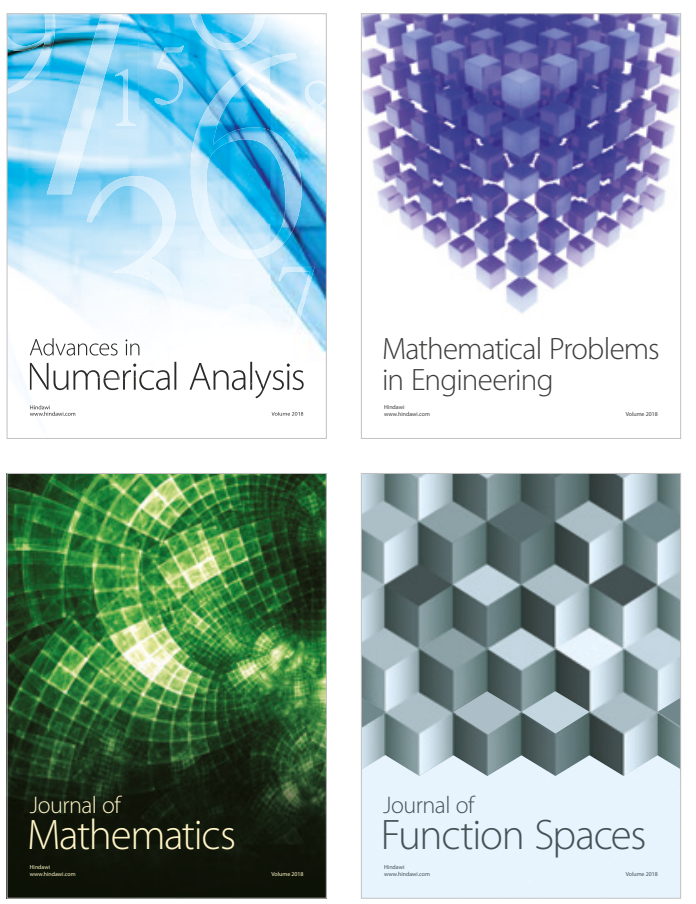

Mathematical Problems in Engineering

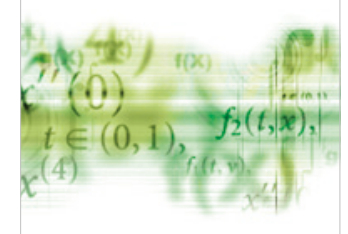

International Journal of

Differential Equations

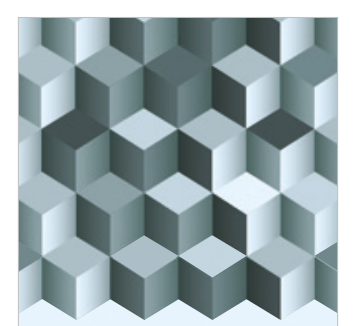

Journal of

Function Spaces

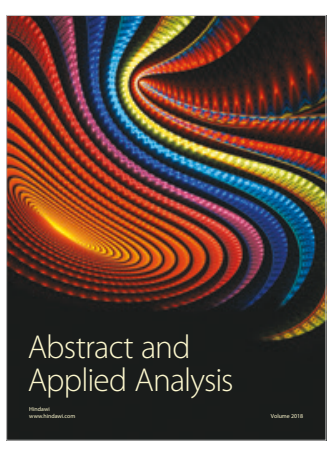

The Scientific

World Journal

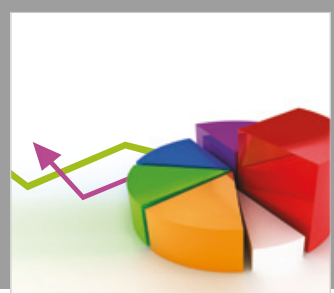

Journal of

Probability and Statistics
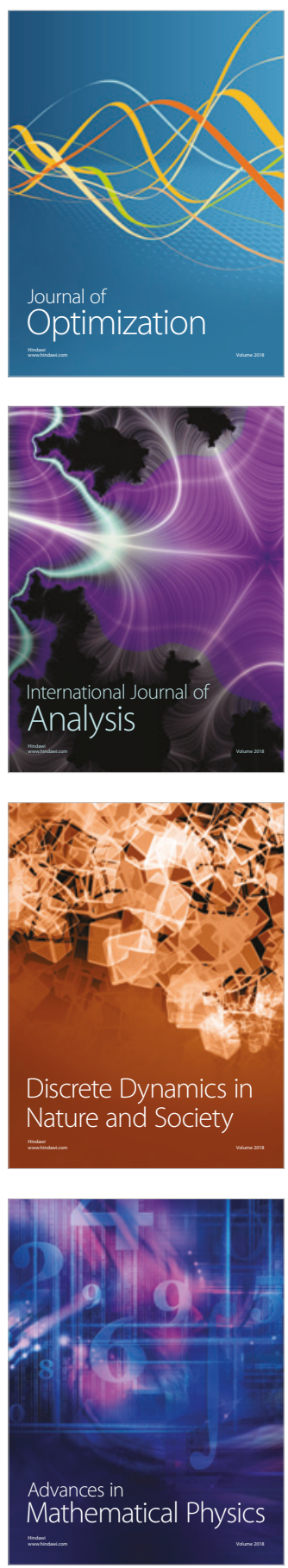